\title{
Evaluación de la Calidad de Vida utilizando el test EORTC QLQ C15-PAL en pacientes en tratamiento del dolor con Morfina Intratecal.
}

\section{Evaluation of Quality of Life using the Eortc Qlq C15-Pal test in patients undergoing treatment of pain with Intrathecal Morphine.}

*Correspondencia: mcvallejom@hotmail.es Teléfono [593] 043718700

Conflicto de intereses: Los autores declaran no tener conflictos de intereses.

Fondos: Ver la página 80

Recibido: 23 Diciembre 2016 Aceptado: 20 Mayo 2017 Publicado: 30 Abril 2018

Membrete bibliográfico: Tomalá $\mathrm{M}$, Vallejo $\mathrm{M}$, Lino $\mathrm{N}$. Evaluación de la calidad de vida utilizando el test EORTC QLQ C15PAL en pacientes en tratamiento del dolor con morfina intratecal. Rev. Oncol. Ecu 2018;28(1):72-83.

DOI: https://doi.org/10.33821/239

Copyright Tomalá, et al. Este artículo es distribuido bajo los términos de Creative Commons Attribution License, el cual permite el uso y redistribución citando la fuente y al autor original.

\section{Marcelo Tomalá1, Mariana Vallejo Martínez²* (D), Nancy Lino².}

1. Postgrado de medicina Interna de la Universidad de Especialidades Espíritu Santo, Guayaquil-Ecuador.

2. Servicio de Dolor y cuidados Paliativos del Instituto Oncológico Nacional "Dr. Juan Tanca Marengo"-SOLCA-Guayaquil, Ecuador.

\section{Resumen}

Introducción: El manejo del dolor mediante dispositivos intratecales con morfina es una opción de tratamiento de cuidados paliativos en pacientes oncológicos. El objetivo del presente estudio fue determinar la calidad de vida en un grupo de pacientes que utilizan esta opción de tratamiento.

Métodos: El presente estudio observacional se desarrolló en el Servicio de Dolor y Cuidados Paliativos del Hospital de SOLCA - Guayaquil. Se midió la calidad de vida con el cuestionario EORTC QLQ-C15PAL a los 0,30 y 90 días en un grupo de pacientes con cáncer terminal y dolor oncológico que fueron tratados con analgesia intratecal por medio de un dispositivo multiprogramable, usando morfina. El período de estudio fue enero a diciembre del 2015, los pacientes firmaron un consentimiento informado. Se compara la calidad de vida usando la prueba Kruskal-Wallis.

Resultados: Se incluyeron en el estudio 15 pacientes, 10 mujeres (66.7\%), con edad media de $50 \pm 15$ años. La localización de cáncer más frecuente fue colo-rectal 5 casos $(33 \%)$. La calidad de vida global al día 0 fue de $27.6 \pm 4$ puntos, al día 30 fue de $90.7 \pm 10$ puntos $(P<0.001)$ y al día 90 fue de $88.2 \pm 10$ puntos $(\mathrm{P}<0.001)$. A los 90 días, el estado funcional disminuyó 57.5 puntos, el estado emocional -60.5 puntos, Disnea -50.3 puntos, Dolor -71 puntos, Insomnio -77.9 puntos, Fatiga -64 puntos, Apetito -53.7 puntos, Nauseas -41.9 puntos, Constipación -56.8 puntos.

Conclusión: La analgesia intratecal con morfina en 15 pacientes con cáncer terminal, mejora la calidad de vida de $27.6 \%$ a $88.2 \%$ en un lapso de 3 meses y la calidad de vida se mantiene hasta 90 días. 
Palabras Claves: CALIDAD DE VIDA, DOLOR EN CÁNCER, ANALGESIA CONTROLADA POR EL PACIENTE, CUIDADO TERMINAL, MANEJO DEL DOLOR, CUIDADOS PALIATIVOS.

DOI: $10.33821 / 239$

\section{Abstract}

Introduction: Pain management through intrathecal devices with morphine is an option for the treatment of palliative care in cancer patients. The aim of the present study was to determine the quality of life in a group of patients who use this treatment option.

Methods: The present observational study was developed in the Pain and Palliative Care Service of the Hospital of SOLCA - Guayaquil. Quality of life was measured with the EORTC QLQ-C15-PAL questionnaire at 0,30 and 90 days in a group of patients with terminal cancer and oncological pain who were treated with intrathecal analgesia using a multiprogrammable device, using morphine. The study period was from January to December 2015, patients signed an informed consent. The quality of life is compared using the Kruskal-Wallis test.

Results: Fifteen patients were included in the study, 10 women (66.7\%), with an average age of $50 \pm$ 15 years. The most frequent cancer localization was colo-rectal 5 cases (33\%). The overall quality of life at day 0 was $27.6 \pm 4$ points, at day 30 it was $90.7 \pm 10$ points $(P<0.001)$ and at day 90 it was 88.2 \pm 10 points $(P<0.001)$. At 90 days, the functional state decreased 57.5 points, the emotional state 60.5 points, Dyspnea -50.3 points, Pain -71 points, Insomnia -77.9 points, Fatigue - 64 points, Appetite -53.7 points, Nausea -41.9 points, Constipation -56.8 points.

Conclusion: The intrathecal analgesia with morphine in 15 patients with terminal cancer, improves the quality of life from $27.6 \%$ to $88.2 \%$ in a period of 3 months and the quality of life is maintained up to 90 days.

Keywords: QUALITY OF LIFE, PAIN IN CANCER, ANALGESIA CONTROLLED BY THE PATIENT, TERMINAL CARE, PAIN MANAGEMENT, PALLIATIVE CARE.

DOI: $10.33821 / 239$

\section{Introducción}

La Organización Mundial de la Salud define la Calidad de Vida (CV) como «la percepción que un individuo tiene de su lugar en la existencia, en el contexto de la cultura y del sistema de valores en los que vive y en relación con sus expectativas, sus normas y sus inquietudes.»[1], por lo que se reconocen diferentes aspectos dentro del concepto general entre los cuales tenemos: El bienestar físico, psicológico-social, el entorno y el nivel de independencia. La CV por lo tanto es un concepto dual que integra aspectos subjetivos en relación a la sensación de calma del paciente y objetivos asociado al bienestar según los cuidados brindados.

El término de CV se emplea actualmente en diferentes campos del sistema de salud, pero no tiene una definición aceptada universalmente, en el campo de la oncología este término se centra en la salud del paciente con enfermedad oncológica y progresión de su enfermedad que genera un pronóstico reservado con múltiples síntomas, en primera línea 
el dolor [2]. El dolor en los pacientes con cáncer, es un síntoma de alta prevalencia que deteriora de forma importante su CV. Entre el 30-50\% de los pacientes presentan dolor en el momento del diagnóstico de una neoplasia, y en las fases avanzadas puede llegar al 70 $90 \%$ [3], siendo este secundario, a progresión de la enfermedad o relacionada con los tratamientos y/o patologías asociadas.

La evaluación de CV se expresa en forma de índices globales o multidimensionales por lo que existen instrumentos para su medición, En 1981 se diseñó un cuestionario específico para evaluar la calidad de vida de los pacientes con cáncer; la EORTC por las siglas en inglés ( European Organization for Research and treatment of Cancer) escala QLQ-C30; cuestionario especifico de la enfermedad y auto cumplimiento que mide el impacto de la enfermedad y del tratamiento sobre la salud física, los síntomas psicológicos y el funcionamiento social en los pacientes con cáncer. Existe una versión reducida que es específicamente para medicina paliativa [4] el QLQ-C15-PAL [5]. Esta escala consta de 15 puntos, que puede ser complementada por un observador.

Para el manejo del dolor por cáncer las opciones de abordaje son integrales, como dolor total ayudándose de instrumentos que permitan objetivar la funcionabilidad, el componente emocional y social que se apoya con el uso de los cuestionarios. EL tratamiento con opioides es el método estándar para el control del dolor oncológico crónico. Se conoce ampliamente que aproximadamente el $90 \%$ de los pacientes afectados con cáncer en fase terminal van a precisar de ellos para el alivio de su dolor [6]. Ejerciendo sus efectos al imitar sustancias naturales, llamadas péptidos opioides endógenos o endorfinas, su uso es por varias vías ya sea oral, parenteral e intratecal [7]. Produciendo efectos farmacológicos al interactuar con los receptores opioides, acoplados a proteínas G. Los receptores opioides identificados son: $\mu, \kappa, y \delta$. El mecanismo de acción se basa en una marcada y selectiva inhibición de las pequeñas fibras $A$, delta y $C$, las cuales están directamente involucradas en la conducción de la sensación de dolor [8].

Existe un porcentaje 5 - $10 \%$ de pacientes con dolor oncológico crónico complejo que suelen requerir dosis importantes de opioides con efectos adversos relevantes que condicionan la utilidad de la vía intratecal.

La administración de morfina intratecal permite una mayor concentración del fármaco en proximidad con los receptores opioides, ejerciendo el efecto analgésico con dosis significativamente menores que con la vía oral o sistémica, con una potencial reducción de los efectos secundarios de igual manera presenta menores complicaciones que la utilización de catéter epidural [9].

El objetivo del presente estudio fue describir la calidad de vida de los pacientes sometidos a tratamiento de dolor de origen oncológico con una vía intratecal.

\section{Materiales y Métodos}

Se realizó un estudio prospectivo observacional en el Servicio de Dolor y Cuidados Paliativos del hospital de SOLCA - Guayaquil, sobre calidad de vida en pacientes con 
cáncer avanzado tras un periodo de seguimiento desde 3 - hasta 12 meses. Estos pacientes recibieron analgesia intratecal por medio de un dispositivo multiprogramable, brindando una terapia analgésica de acuerdo a las necesidades de los pacientes con menos efectos secundarios del opioide, la información fue extraída del archivo del Servicio de Dolor y Paliativos de la Institución.

El tipo de muestreo fue causal (no probabilístico) de los pacientes que fueron prescritos con dispositivos intratecales para analgesia del dolor. El período de estudio fue enero del 2015 a diciembre del 2015.

La selección de los pacientes se realizó a través de las historias clínicas y fueron entrevistados de manera personal. Se les aseguró su confidencialidad e intimidad al ser ellos las fuentes de información; a los participantes se les entregó un consentimiento informado, explicando el estudio de manera verbal y escrita. Se incluyeron todos los casos posibles de pacientes con diagnóstico de enfermedad oncológica avanzada, de ambos sexos, con edades comprendidas entre los 20 y 70 años, con dolor oncológico complejo que presentaban factores de mal pronóstico para un buen control del dolor (con pobre respuesta a tratamientos convencionales y/o presencia de efectos secundarios relevantes, con pronóstico de vida no inferior a tres meses, diagnósticos oncológicos diversos. Se excluyeron aquellos con trastornos de la coagulación, estado nutricional deficiente, infección local o sistémica, deformidad de la columna que limitara la colocación del catéter y aquellos que no firmaran el consentimiento informado.

Se utilizó el cuestionario EORTC QLQ-C15-PAL para medir la calidad de vida en 3 periodos de tiempo: al inicio de la terapia analgésica intratecal, a los 30 días y a los 90 días. En el estudio del dolor como parámetro principal dentro de la valoración de estos pacientes se procedió a hacer uso de la escala visual análoga (EVA) en 3 fases: al inicio, en los controles y al final del estudio.

En el análisis estadístico se describieron las variables con frecuencias y porcentajes para las variables categóricas. Para las variables en escala se describe el promedio y la desviación estándar. Los promedios de CV se compararon con la prueba Kruskal-Wallis. Los valores $\mathrm{P}$ se ajustaron con el método de Bonferroni. El paquete estadístico utilizado fue SPSS 19.0 para PC.

\section{Resultados}

Se incluyeron en el estudio 15 pacientes, 10 mujeres (66.7\%), con edad media de 50 años (35 - 65 años). La localización de cáncer más frecuente fue colon-rectal $33 \%$, seguido por otros tipos de cáncer 6 \% (plasmocitoma, mieloma múltiple, leiomiosarcoma). El Índice de Karnosky al inicio del estudio en la totalidad de los pacientes dio una media de 78 \% (60 90) (Tabla 1). 
Tabla 1. Características de los pacientes.

\begin{tabular}{|l|c|c|}
\hline \multicolumn{2}{|c|}{$\begin{array}{c}\text { Características de Pacientes } \\
n=15\end{array}$} & Datos \\
\hline & & $(\mathrm{DE} 15)$ \\
\hline Edad & 50 & $(20-70)$ \\
\hline Media & 51 & \\
\hline Mediana (años) & & $33 \%$ \\
\hline & & $67 \%$ \\
\hline Sexo & 5 & \\
\hline Masculino & 10 & $13 \%$ \\
\hline Femenino & & $33 \%$ \\
\hline & & $7 \%$ \\
\hline Tipo de Cáncer & 2 & $7 \%$ \\
\hline Próstata & 5 & $40 \%$ \\
\hline Colo-rectal & 1 & \\
\hline Ovario & 1 & $(\mathrm{DE} 16)$ \\
\hline Mama & 6 & $(50-100 \%)$ \\
\hline Otros & \multicolumn{2}{|c|}{} \\
\hline & 78 & \\
\hline Índice de karnosfky (IK) & 80 & \\
\hline Media & & \\
\hline Mediana (\%) & & \\
\hline
\end{tabular}

En la valoración de la CV utilizando EORTC C15-PAL, al inicio del estudio hubo un compromiso importante tanto funcional como emocional en estos pacientes con una media de 87.5 y 81.4 respectivamente. Se trataban de pacientes muy sintomáticos, entre los cuales las molestias predominantes fueron el insomnio (98.7\%), el dolor (97.7\%) Estos pacientes dieron una puntuación en CV global muy baja (27.6\%).

Re-evaluando a los pacientes a los 30 días de colocación de morfina Intratecal (IT), existieron cambios positivos con una reducción importante del dolor (26.7\%), obteniendo como CV global (88.2 \%) que representa una mejoría relevante en la calidad de vida manteniéndose estable en su sintomatología durante 3 a 6 meses y luego decayendo en el $50 \%$ de los pacientes presentados debido a la constante actividad tumoral que avanza pero con buen manejo de su dolor con dosis bajas de morfina intratecal (Tabla 2).

En lo que respecta a la valoración de la intensidad del dolor, se obtuvo un EVA con una media de 9 previo a la instauración de la bomba intratecal de morfina, al final del estudio se obtuvo un EVA con una media de 1. 
Tabla 2. Comparación de la media del score de EORTC QLQ C15-PAL en diferentes periodos de tiempo incluir escala EORTC QLQ C15-PAL.

\begin{tabular}{|c|c|c|c|c|c|c|c|c|}
\hline \multirow[t]{2}{*}{ EORTC QLQ C15-PAL } & \multicolumn{2}{|c|}{ Día 0} & \multicolumn{2}{|c|}{ Día 30} & \multicolumn{2}{|c|}{ Día 90} & \multicolumn{2}{|c|}{$P$} \\
\hline & \multicolumn{2}{|c|}{$n=15$} & \multicolumn{2}{|c|}{$n=15$} & \multicolumn{2}{|c|}{$n=12$} & $\begin{array}{l}\text { Día } 0 \\
\text { vs } 30\end{array}$ & $\begin{array}{c}\text { Día } \\
30\end{array}$ \\
\hline Estado funcional & 87.5 & \pm 11 & 29.8 & \pm 10 & 30.0 & \pm 10 & $<0.001$ & 0.94 \\
\hline Estado emocional & 81.4 & \pm 16 & 21.0 & \pm 6 & 20.9 & \pm 6 & $<0.001$ & 0.81 \\
\hline Disnea & 72.0 & \pm 13 & 19.3 & \pm 3 & 21.7 & \pm 6 & $<0.001$ & 0.77 \\
\hline Dolor & 97.7 & \pm 7 & 26.0 & \pm 9 & 26.7 & \pm 9 & $<0.001$ & 0.99 \\
\hline Insomnio & 98.7 & \pm 4 & 20.7 & \pm 3 & 20.8 & \pm 3 & $<0.001$ & 1.0 \\
\hline Fatiga & 89.4 & \pm 14 & 25.7 & \pm 15 & 25.4 & \pm 13 & $<0.001$ & 1.0 \\
\hline Apetito & 78.7 & \pm 12 & 24.0 & \pm 8 & 25.0 & \pm 9 & $<0.001$ & 0.90 \\
\hline Nauseas & 62.7 & \pm 10 & 20.0 & \pm 4 & 20.8 & \pm 3 & $<0.001$ & 0.99 \\
\hline Constipación & 79.3 & \pm 3 & 21.3 & \pm 5 & 22.5 & \pm 6 & $<0.001$ & 0.89 \\
\hline QLQ global & 27.6 & \pm 4 & 90.7 & \pm 10 & 88.2 & \pm 10 & $<0.001$ & 0.78 \\
\hline
\end{tabular}

\section{Discusión}

Este reporte describe la mejoría de la calidad de vida a los 30 días en los pacientes que fueron sometidos al tratamiento intratecal para el control del dolor. Esta diferencia de mejoría en la calidad de vida fue de 63 puntos a los 30 días y 61 puntos a los 90 días del tratamiento con analgesia intratecal. Existen varios estudios $[10,11]$ sobre la utilización de la escala EORTC QLQ C15-PAL que demuestran la validez para medir la calidad de vida mediante tres parámetros: el estado funcional en relación a los síntomas, la esfera emocional y la percepción global de su escala de vida, que coinciden en que esta escala es un recurso muy importante en la valoración de los pacientes valorados en cuidados paliativos. En estos estudios la población estudiada fue mayormente del género masculino lo que difiere de la presente muestra. La edad de los estudios reportados fue mayor a 59 años, el síntoma peor tolerado fue la fatiga (75.9), al contrario en el presente reporte fue el dolor y el insomnio con 97.7 y 98.7 puntos sobre 100. La calidad de vida percibida tuvo una media 37.3 en los estudios referidos, sin embargo en el presente estudio fue 10 puntos menos (27.6).

Un estudio multicéntrico [12] realizado en Francia, Italia, Alemania, España y Taiwán se incluyeron las características sociodemográficas tanto en manejo ambulatorio como atención sanitaria, sus resultados indicaron la población mayormente afectada fueron hombres entre 50 y 60 años, se encontraron diferencias significativas $(P<0.001)$ entre grupos geoculturales por edad, nivel de educación, género, configuración del barrio, toxicidad del tratamiento, estado de salud global, número de enfermeras por cama, número 
de médicos por cama y tamaño del hospital con lo que se concluyó que no existe relación entre la calidad de vida percibida con la satisfacción dada por la asistencia sanitaria.

Otro estudio en España [13] coincide en que los pacientes con mayor frecuencia constituyen los hombres con una edad media de 64 años, el síntoma mejor controlado fue la disnea obteniendo 34.8 puntos de media y el peor controlado con 53.2 puntos de media fueron las náuseas y vómitos. La afectación funcional fue tolerable (33.3) y el componente emocional mayormente afectado (51.4). La CV percibida se consideró buena con una media de 51.7, se difiere del estudio presente por la gran variedad de neoplasias incluidas y no todos con las intenciones de manejo del dolor como en este reporte.

En reportes en China [14] y Canadá [15], con 243 y 364 pacientes respectivamente, clasifican a los pacientes por grupos según ECOG-PS. Difieren de otros estudios en cuanto al género que en este caso el de mayor frecuencia fue el sexo femenino (56.2 \%) y (78.3\%) respectivamente. Estos estudios llegaron a la conclusión de que a medida que aumenta, los puntajes para la función física, la función emocional y calidad de vida global disminuye, mientras que las puntuaciones para las dimensiones de los síntomas se mantienen variables pero en incremento.

Otros dos estudios en Turquía [16], México [17] y Brasil [18] valoraron la CV en grupos de pacientes según la puntuación asignada por el Índice de Karnofsky con una población promedio de 100 participantes, cuyas edades dieron un promedio de 54 años. En los tres estudios predomino el sexo femenino. Con respecto a la valoración funcional, sintomática y calidad de vida percibida se observaron mejores puntajes de salud global mientras más alto sea el puntaje de Karnofsky; de esta manera concluyendo que existe mejor de calidad de vida asociado a una mejor función física y un mejor control sintomático.

En el presente estudio el sexo femenino fue más prevalente. La edad promedio se ha mantenido a nivel mundial entre 50 a 60 años. Nuestros pacientes dieron una puntuación en EORTC QLQ C15-PAL tanto funcional, sintomática y global alta al inicio del estudio lo que coincide con otros autores en sus conclusiones: a mayor afectación sintomática y limitación funcional; menor calidad de vida. Existió una mejoría en todos los scores luego de la aplicación de morfina intratecal no solo controlando el dolor sino además otros síntomas y beneficiando al estado físico y emocional.

En este grupo de pacientes el dolor era el mayor componente, siendo este el factor generador de otras afecciones con cambios en el estado funcional, y que no existía respuesta ante tratamientos convencionales. El uso de morfina intratecal mejora la calidad de vida y puede tener otros beneficios como la disminución de los efectos secundarios de adicción, estreñimiento grave, debido a que se requieren menos dosis del opioide, adicionalmente se demuestra que el tratamiento analgésico opioide intratecal colaboró en el control y estabilidad de los síntomas asociados que generan sufrimiento y desmejoran la calidad de vida de los pacientes con enfermedades terminales.

Desde la perspectiva de la medicina paliativa, el concepto de "calidad de vida" se incluye la posibilidad de vivir experiencias gratificantes, sea cual fuere la situación funcional, tomando en cuenta el tratamiento del dolor. El concepto de calidad de vida relacionada con 
la salud es un fenómeno subjetivo multidimensional que se presta a diversas interpretaciones, desde el bienestar, placer y comodidad hasta la dignidad de la persona.

El presente estudio como todo estudio observacional tiene sus limitaciones, iniciando por el número de pacientes incluidos en el estudio, el cual es la casuística de un centro oncológico de pacientes que reciben analgesia intratecal. Otra limitación fue la selección de un grupo control y mediciones de variables clínicas relacionadas al dolor de tipo oncológico. En próximas investigaciones controladas se deberán responder estas interrogantes.

\section{Conclusiones}

La analgesia intratecal con morfina en 15 pacientes con cáncer terminal, mejora la calidad de vida de $27.6 \%$ a $88.2 \%$ en un lapso de 3 meses, permite un control del dolor de EVA 9 (Día cero) a EVA 1 (Día 90).

\section{Agradecimientos}

Se reconoce a las personas que participaron indirectamente en el estudio tales como los pacientes, como personal técnico, otras en general de ION "Dr. Juan Tanca Marengo" SOLCA-Guayaquil.

\section{Información adicional}

Nota del Editor

La Revista Oncología Ecu permanece neutral con respecto a los reclamos jurisdiccionales en mapas publicados y afiliaciones institucionales.

\section{Abreviaturas}

CV: Calidad de vida.

EVA: Escala de Valoración Analgésica.

EORTC: European Organization for Research and treatment of Cancer. QLQ C15-PAL: Quality of Live of 15 questions about palliative care. ECOG-PS: Eastern Cooperative Oncology Group Performance Status.

\section{Archivos Adicionales}

Ninguno declarado por los autores.

\section{Fondos}

Los fondos para la presente investigación fueron propios de los autores del presente artículo. 


\section{Disponibilidad de datos y materiales}

Existe la disponibilidad de datos bajo solicitud al autor de correspondencia. No se reportan otros materiales.

\section{Contribuciones de los autores}

MT, MV, NL realizaron la idea de investigación, diseño y el análisis crítico del artículo. MT realizó la recolección de datos, análisis estadístico y la escritura académica, Todos los autores leyeron y aprobaron el manuscrito final.

Aprobación de ética y consentimiento para participar

El estudio fue aprobado por el comité local, el consentimiento informado para participación en el estudio para cada paciente fue en forma escrita y firmada.

\section{Consentimiento para publicación}

Se obtuvo el consentimiento firmado para publicación de los datos en el presente estudio por parte de los participantes.

\section{Información de los autores}

Marcelo Tómala, medico postgradista de medicina Interna de la Universidad de Especialidades Espíritu Santo, Guayaquil-Ecuador.

Mariana Vallejo Martínez, médica tratante del Servicio de Dolor y cuidados Paliativos del Instituto Oncológico Nacional "Dr. Juan Tanca Marengo"-SOLCA-Guayaquil, Ecuador. https://orcid.org/0000-0001-5727-262X

Nancy Lino, médica tratante del Servicio de Dolor y cuidados Paliativos del Instituto Oncológico Nacional "Dr. Juan Tanca Marengo"-SOLCA-Guayaquil, Ecuador.

Revisiones por pares

Acceda a la revisión de pares académicos en el siguiente enlace: https://publons.com/review/2770191 


\section{Referencias}

Abreviaturas en la referencias

DOI: Digital Object

Identifier

PMID: PubMed Identifier

SU: Short URL
1. WHO [Internet]. Geneva: World Health Organization; c2005-2015 [cited 2016 Oct 22]. Available from: http://www.who.int/healthinfo/survey/whoqol-qualityoflife/en/

2. González-Escalada J, Camba A, Casas A, Gascón P, Herruzo I, Núñez-Olarte J, et al. Código de buena práctica para el control del dolor oncológico. Med Paliat 2011;18(2):63-79. DOI: 10.1016/S1134$\underline{248 \times(11) 70011-8}$

3. Puebla Díaz F. Tipos de dolor y escala terapéutica de la O.M.S. Dolor iatrogénico. Oncología, 2005; 28 (3):139-143. SU: goo.gl/qPay9h

4. McMillan SC, Small BJ. Symptom distress and quality of life in patients with cancer newly admitted to hospice home care. Oncol Nurs Forum 2002; 29 (10):1421-1428. DOI: 10.1188/02.0NF.1421-1428

5. Castañeda-de-la-Lanza C, O'Shea G, Narváez M, Lozano J, Castañeda-Pena G, Castañeda-de la-Lanza J. Calidad de vida y control de síntomas en el paciente oncológico. Gaceta Mexicana de Oncología. 2015;14(3):150-156. DOI: 10.1016/j.gamo.2015.07.007

6. Benítez MA, Pérez Suárez MC, Fernández Díaz R, Cabrejas Sánchez A. Cuidados Paliativos. Tratamiento del dolor oncológico crónico (II): el uso de los opiáceos. Aten Primaria 2002;29:513-6. SU: goo.gl/vna7YX

7. Goodman \& Gillman. Anestésicos Opioides. Las bases farmacológicas de la terapéutica. Novena edición. México: Mc GrawHill, 2004:737-748.

8. Casati A, Vinciguerra F. Intrathecal anestesia. Curr Opin Anaesthesiol 2002;15:543-551. SU: goo.gl/CzULiN

9. Vaquerizo-García V, García-López M, Plasencia-Arriba MA, Maestre-García C. Control del dolor postoperatorio mediante morfina intratecal en pacientes intervenidos por fusión vertebral instrumentada. Acta Ortopédica Mexicana 2015; 29(1):34-39. DE: goo.gl/p6jD6D

10. Kool K, Kerba M, Zeng L, Zhang L, Chen E, Chow E, et al. Quality of life in patients with brain metastases receiving upfront as compared to salvage stereotactic radiosurgery using the EORTC QLQ-C15-PAL and the EORTC QLQ BN20 + 2: a pilot study. J Radiat Oncol. 2013;2:217-24. DOI: 10.1007/s13566-012-0052$\underline{9}$

11. Hermann K, Engeser P, Szecsenyi J, Miksch A. Palliative patients cared for at home by PAMINO-trained and other GPs-healthrelated quality of life as measured by QLQ-C15-PAL and POS. BMC Palliat Care. 2012;11:1-7. DOI: 10.1186/1472-684X-11-13

12. Brédart A, Coens $C$, Aaronson N, Chie WC, Efficace F, Conroy $T$, et al. Determinants of patient satisfaction in oncology settings from European and Asian countries: Preliminary results based on the EORTC INPATSAT32 questionnaire. Eur J Cancer Care. 2007;43:323-30. DOI: 10.1016/j.ejca.2006.10.016

13. Hernández-Belloa E, Bueno-Lozano M, Salas-Ferrer P, Sanz-Armunia M. Percepción de la calidad de vida y satisfacción con la asistencia sanitaria recibida en un grupo de pacientes oncológicos terminales que reciben cuidados paliativos en un hospital de Zaragoza, España. Rev Colomb Cancerol. 2016;20(3):110-116. DOI: 10.1016/j.rccan.2016.04.003.

14. Zhang L, Wang N, Zhang J, Liu J, Luo Z, Sun W, et al. Cross-cultural verification of the EORTC QLQ-C15PAL questionnaire in mainland China. Palliat Med 2016;30(4)401-408. DOI: $10.1177 / 0269216315593671$.

15. Lam K, Zeng L, Zhang L, Tseng LM, Hou MF, Fairchild A, et al. Predictive Factors of Overall Well-Being Using the EORTC QLQ-C15-PAL Extracted from the EORTC QLQ-C30. Journal of Palliative Medicina 2013;16(4): 402-8. DOI: 10.1089/jpm.2012.0398.

16. Ozcelik H, Guzel Y, Sonmez E, Aksoy F, Uslu R. Reliability and validity of the Turkish version of the EORTC QLQ-C15-PAL for patients with advanced cancer. Palliative and Supportive Care 2016;14:628-634. DOI: $10.1017 /$ S1478951516000195 
17. Suárez-del-Real $Y$, Allende-Pérez S, Alférez-Mancera A, Rodríguez R, Jiménez-Toxtle S, Mohar A, Oñate-Ocaña L. Validation of the Mexican-Spanish version of the EORTC QLQ-C15-PAL questionnaire for the evaluation of healthrelated quality of life in patients on palliative care. Psycho-Oncology 2011;20(8):889-896. DOI: 10.1002/pon.1801

18. Nunes NA. The quality of life of Brazilian patients in palliative care: validation of the European Organization for Research and Treatment of Cancer Quality of Life Questionnaire Core 15 PAL (EORTC QLQ-C15-PAL). Support Care Cancer 2014;22(6):1595-600. DOI: 10.1007/s00520-014-2119-1 